\title{
A Head-Mounted Spectacle Frame for the Study of Mouse Lens-Induced Myopia
}

\author{
Yangshun Gu, ${ }^{1}$ Baisheng Xu, ${ }^{1}$ Chunfei Feng, ${ }^{2}$ Yang Ni, ${ }^{3}$ Qin Wu, \\ Chixin Du, ${ }^{1}$ Nan Hong, ${ }^{1}$ Peng Li, ${ }^{3}$ Zhihua Ding, ${ }^{3}$ and Bo Jiang ${ }^{1}$ \\ ${ }^{1}$ Department of Ophthalmology, First Affiliated Hospital, College of Medicine, Zhejiang University, Hangzhou, \\ Zhejiang 310003, China \\ ${ }^{2}$ Department of Operation Room, First Affiliated Hospital, College of Medicine, Zhejiang University, Hangzhou, \\ Zhejiang 310003, China \\ ${ }^{3}$ State Key Lab of Modern Optical Instrumentation, Department of Optical Engineering, Zhejiang University, Hangzhou, \\ Zhejiang 310027, China
}

Correspondence should be addressed to Bo Jiang; drjiangbo@hotmail.com

Received 1 December 2015; Accepted 27 December 2015

Academic Editor: Terri L. Young

Copyright (C) 2016 Yangshun Gu et al. This is an open access article distributed under the Creative Commons Attribution License, which permits unrestricted use, distribution, and reproduction in any medium, provided the original work is properly cited.

\begin{abstract}
The mouse model has been widely employed to explore the mysteries of myopia. For now, existing techniques for induction of experimental myopia in mice can be classified into three types: (1) devices directly glued to the fur; (2) devices attached using a combination of glue and sutures; (3) devices attached using a skull-mounted apparatus. These techniques each have its advantages, disadvantages when considering the devices stability, safety, complexity, effectiveness, and so forth. Thus, techniques for myopia induction in mice have yet to be further refined to popularize the applications. In this pilot study, we introduce a new head fixation device named the head-mounted spectacle frame apparatus for the study of mouse lens-induced myopia. Surgical procedures for device attachment were relatively simple and easy to learn in our study. Effective myopia induction was validated by retinoscopy refraction and axial length measurement using optical coherence tomography. In addition, it showed improved compliance and reliable safety when compared to the published methods. The head-mounted spectacle frame apparatus provides a new choice for the study of lens-induced myopia in mouse. It also allows for the use of form deprivation, making it attractive for future experimental mouse myopia trials.
\end{abstract}

\section{Introduction}

For the past half century, myopia has emerged as an extremely important health issue and the related literature has increased exponentially $[1,2]$. And epidemiological data suggested that the prevalence and incidence of myopia were increasingly high, causing serious social and economic burdens [3]. So both scientists and ophthalmologists showed great interest and applied significant effort to elucidate the mechanism of myopia development.

As an important component in the field of myopia research, animal model has greatly expanded our knowledge on the visual regulation of refractive development $[4,5]$. These animal models such as monkeys, chicks, tree shrews, fish, and guinea pigs each have its advantages and disadvantages [6-8]. However, presently consensus has not been established yet regarding which model is ideal. Recently, the establishment of mouse model of myopia has contributed to a recent surge in interest and revealed several essential findings [9-11]. The mouse model offers a highly efficient model in which to study the genetic and environmental basis of the growth of the eye, as well as gene-environment interactions [12-14]. Nevertheless, available techniques for device attachment in mice are limited. In methods using sutures, glue, or a combination of techniques, the goggle or defocusing lens may be easily scratched off and lead to poor ocular health $[11,15,16]$. Other researchers introduced an Elizabethan collar to avoid troublesome scratches, but 


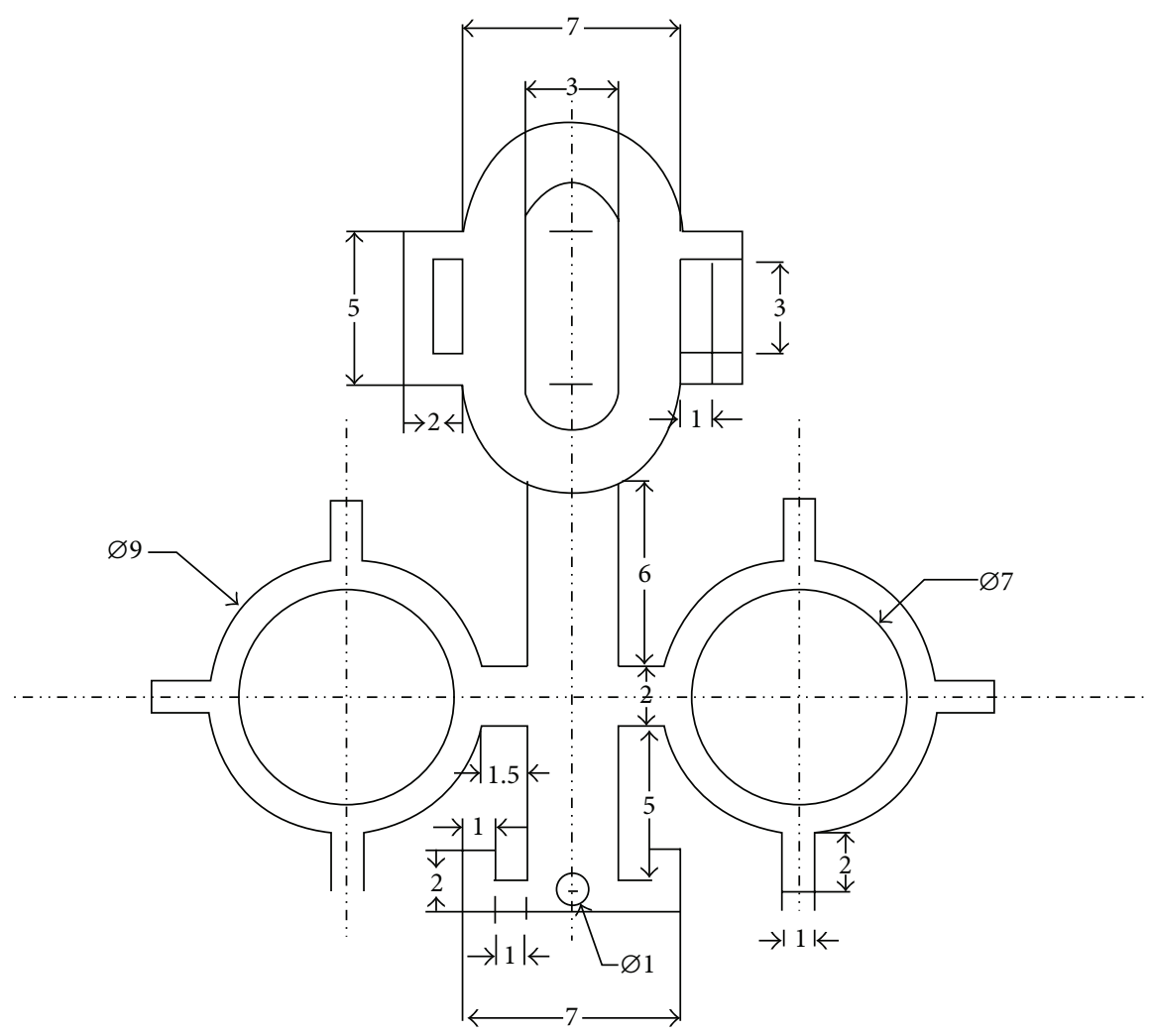

FIGURE 1: The physical dimensions for the frame (unit: $\mathrm{mm}$ ).

the collar may seriously impact the animal's activity and systemic health $[11,17]$. The head-mounted goggle apparatus was reported to be highly stable and efficient. However, the components of goggle apparatus were not convenient fabrication and the surgical procedures were complicated [18].

Then, the purpose of this study is to introduce a simple head fixation device for myopia induction in mice that (1) can be attached with simplified procedures, (2) should keep stable for a certain period of time, (3) effectively induce experimental myopia, and (4) is reliably secured to the eye and whole health.

\section{Materials and Methods}

2.1. Design of the Head Fixation Device. The head fixation device is composed of two parts, the nylon connector and the metal spectacle frame. The nylon connector, including $\mathrm{M} 3 \times 8$ nylon screws, gaskets, and caps, was designed and produced (Shanghai Zhisheng Plastic Co., Ltd.). The spectacle frames were first designed using Rhinoceros software (Version 5.0, Robert McNeel \& Associates; Figure 1) and then were made of 304 stainless steel by laser cutting (Versolsolar Hangzhou Co., Ltd.). A single spectacle frame has four functional components including the (1) kidney-shaped slotted hole for position control, (2) rounded lens clip for experimental lens fixation, (3) nose pad for strengthening fixation, and (4) connecting rod for integration. The spectacle frames underwent manual shaping to be attached to the mouse head; the physical dimensions and a computer representation of the 3D shape for the frame are shown in Figures 1 and 2.

\subsection{Surgical Procedures}

(1) The mice were anesthetized by intraperitoneal injection of $4 \%$ chloral hydrate $(10 \mathrm{mg} / \mathrm{kg}$, Figure 3(a)).

(2) The dorsal cranial fur was shaven; the surgical area was cleaned with Betadine (Figure 3(b)).

(3) A middle line incision (8-10 mm) was made to expose the dorsal cranial surface of the skull.

(4) The exposed fascia and periosteum were removed from the coronal suture to the sagittal suture, and the surface was cleaned and dried (Figure 3(c)).

(5) The screw was sutured to the bilateral temporal muscle, cervical trapezius muscle, and frontal skin with 6-0 nylon sutures (Figure 3(d)).

(6) The incision was sutured with 6-0 nylon suture, and antibiotic ointments were applied (Figure 3(e)).

(7) The gasket and screw were fixed to supply a platform supporting the spectacle frame (Figure 3(f)).

(8) The spectacle frame was placed on the head, and the cap was screwed in and adjusted to position the lens clip (Figure 3(g))

(9) The fixation was strengthened by suturing the nose pad to the skin, and the experimental lenses were fixed (Figure 3(h)). 


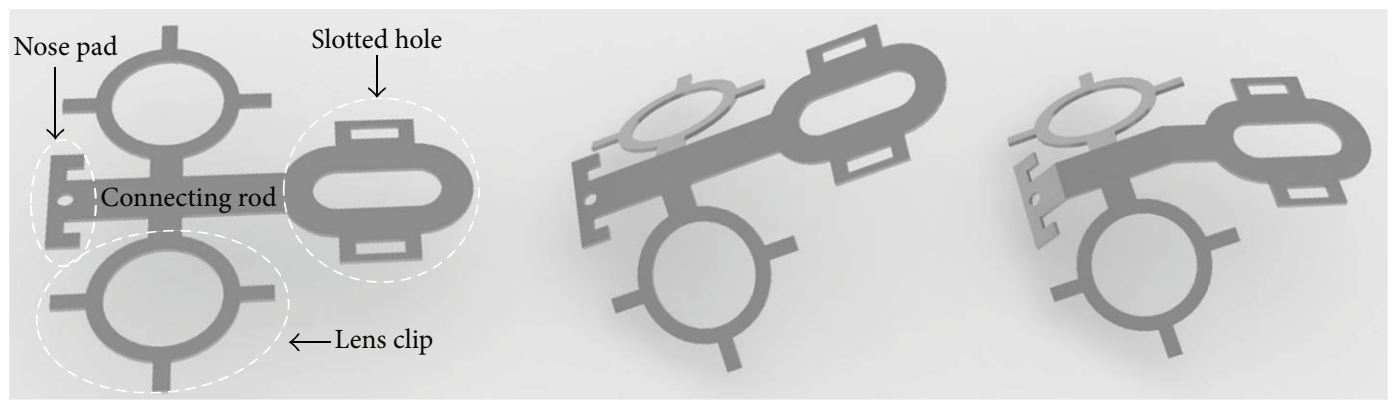

Figure 2: A schematic of the spectacle frame showing the functional components including the slotted hole, lens clip, nose pad, and connecting rod. And a brief visualization of the $3 \mathrm{D}$ shape is present.

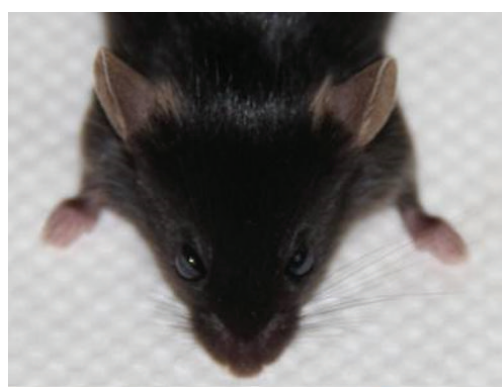

(a)

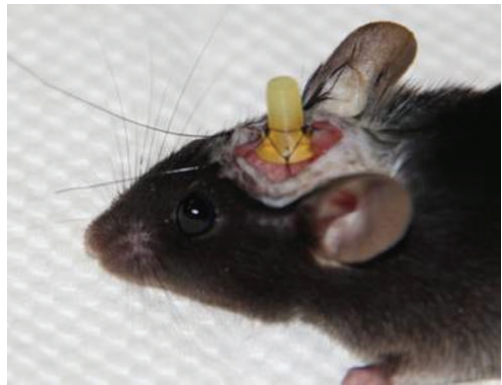

(d)

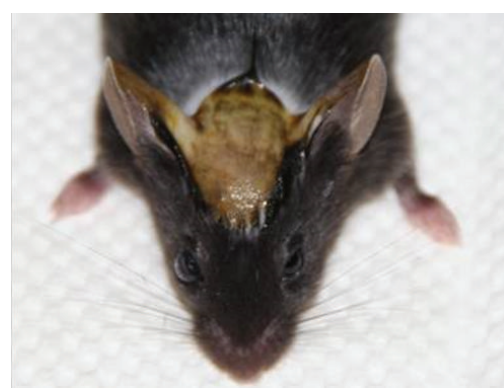

(b)

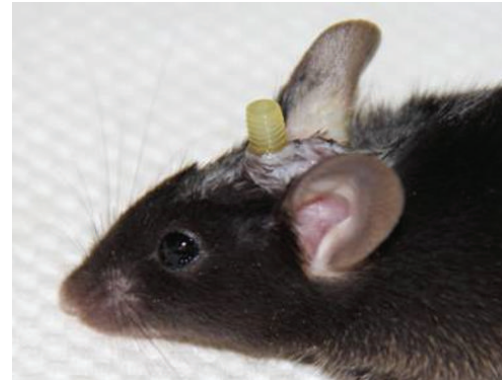

(e)

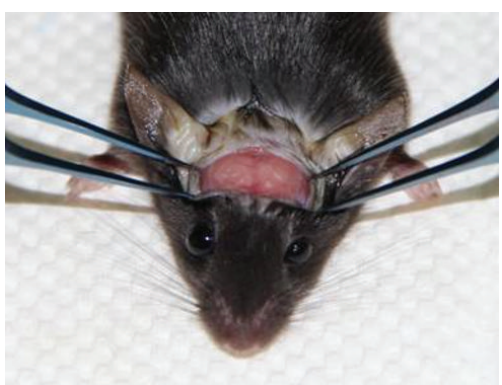

(c)

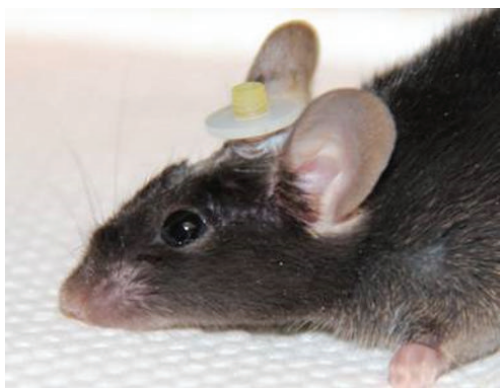

(f)

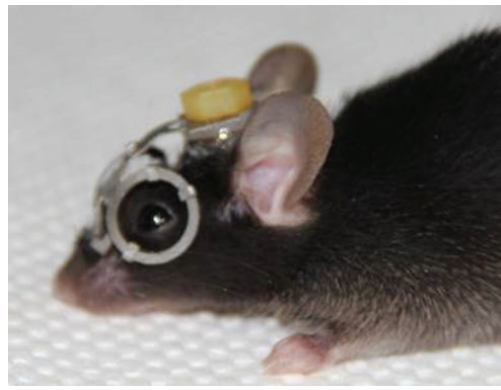

(g)

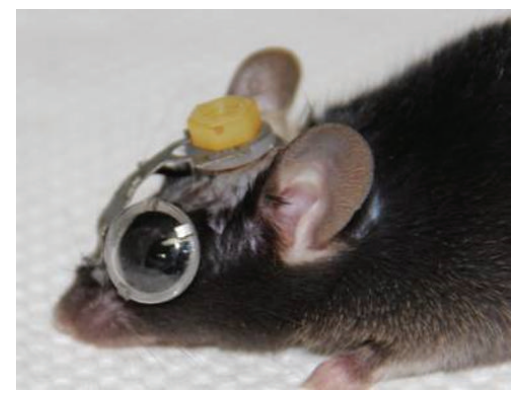

(h)

FIGURE 3: Simplified operation procedures of the head-mounted spectacle frame apparatus: (a) general anesthesia; (b) incision preparation; (c) exposure of the skull and muscle; (d) suturing the screw; (e) suturing the incision; (f) fixing the gasket; (g) fixing the spectacle frame; and (h) fixing the experimental lenses.

2.3. Experimental Design. Male C57BL6J mice aged postnatal 28 days (P28) underwent baseline retinoscopy refraction and axial length measurement using a custom-built optical coherence tomography (OCT), which has been detailed in $[19,20]$ (Figure 4). Two groups were set in the current study.
In the experimental group $(n=25)$, myopia was monocularly induced with the head-mounted spectacle frame over the right eye using a -15.0 diopter (D) lens (Hangzhou Boston Optics Co., Ltd.); no defocusing lens or plano lens was fixed to the left lens clip. In the control group $(n=10)$, no 


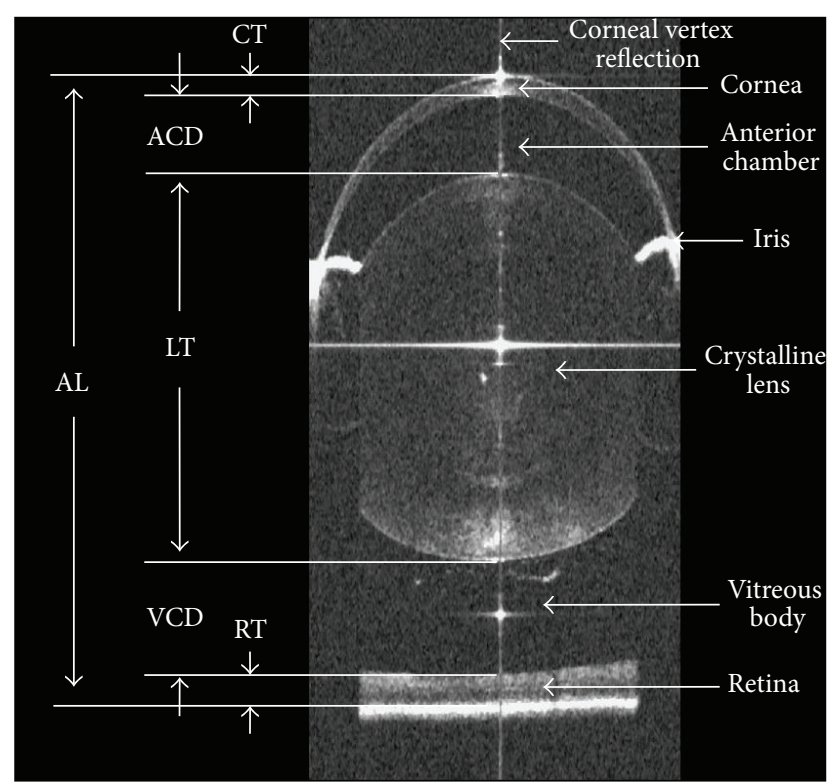

FIGURE 4: The schematic diagram of axial length measurement from the anterior corneal surface to the retinal pigment epithelium along the corneal vertex reflection.

spectacle frame or lens was fixed. The experimental mice were housed in isolation, and the control mice were housed in groups of five. The body weight was measured weekly in experimental group from the time of purchase (P21) to the sacrifice date (P56). All mice were exposed to a $12 \mathrm{~h}$ light: $12 \mathrm{~h}$ dark cycle and checked daily for 28 days to assess the defocusing lens position stability. Then mice aged P56 were refracted and measured again to assess the effectiveness of myopia induction. In addition, a coronal segment of the tissue corresponding to the locations of the underlying screws was serially sectioned and H\&E stained.

2.4. Ethics Statement. This study was approved by the Institutional Animal Care and Use Committee (IACUC) at Zhejiang University (Permit number: Zju201306-1-01-066). All experimental procedures were conducted in accordance with the Guidelines for the Care and Use of Laboratory Animals of Zhejiang University. All mice were sacrificed by an anesthetic overdose, and all efforts were made to minimize suffering.

\section{Results}

There were no intraoperative complications, and none of the animals experienced postoperative incision infection. The mice wearing head-mounted spectacle frames appeared to have normal activity and were well groomed and freely feeding (see video in Supplementary Material available online at http://dx.doi.org/10.1155/2016/8497278). Mice weights were monitored weekly, and weight gain of mice in experimental group was appropriate when compared with the reference values from the JAX LAB (Figure 5).

There were two situations to be noted in the assessment of device stability, a spectacle frame "lost" and an entire

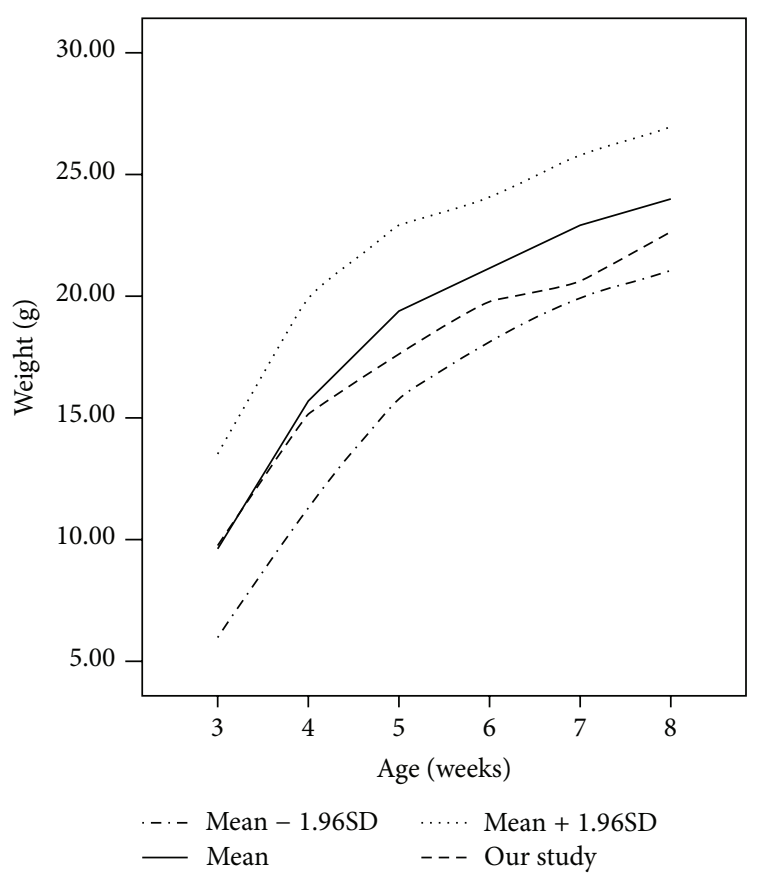

FIGURE 5: A body weight chart for the mice in experimental group. Reference values (mean, mean $\pm 1.96 \mathrm{SD}$ ) were obtained from the JAX LAB (http://jaxmice.jax.org/support/weight/000664.html). The mean weight of mice in experimental group falls between the reference mean and mean $-1.96 \mathrm{SD}$.

apparatus "lost." In these cases, the defocusing lens could flip away from the eye and allowed for some normal visual input, which required timely intervention. The "lost" of spectacle frame was always the result of a loosened screw cap or nose pad and could be retightened in minutes. An entire "lost" apparatus was usually caused by frequent scratching, tissue reaction, or accidental clamping with the cage, and the nylon connector and spectacle frame should be refixated under general anesthesia. In this study (Table 1), 2 cases of spectacle frame loss but no entire apparatus loss were found in the first week (P29-P35), 8 cases of spectacle frame loss and 2 cases of entire apparatus loss occurred in the second week (P36-P42), 12 cases of spectacle frame loss and 3 cases of entire apparatus loss occurred in the third week (P43-P49), and 13 cases of spectacle frame loss and 3 cases of entire apparatus loss occurred in the fourth week (P50-P56). With this technique, $9 / 30$ mice lost the spectacle frame or entire apparatus once, 14/30 mice lost the spectacle frame or entire apparatus twice, and 2/30 lost the spectacle frame or entire apparatus three times during the 28 days induction period. In the first two weeks, no mice lost the spectacle frame or entire apparatus three or more times. Our apparatus stability was relatively superior to the way that directly glued to the fur and was essentially the same as the head-mounted goggling apparatus reported by Faulkner et al. [18] (Table 2).

Refractive error data in Table 3 demonstrated no significant difference between the right and left eyes in the experimental group at P28 $(0.08 \pm 1.26 \mathrm{D}$; paired $t$-test $p=$ $0.75)$ or in the controls $(0.70 \pm 1.34 \mathrm{D}$; paired $t$-test $p=0.13)$. 
TABLE 1: Stability assessment of the head-mounted spectacle frame apparatus.

\begin{tabular}{lccc}
\hline Experimental duration & $\begin{array}{c}\text { \# times } \\
\text { spectacle } \\
\text { frame was } \\
\text { lost }\end{array}$ & $\begin{array}{c}\text { \# times } \\
\text { entire } \\
\text { apparatus } \\
\text { was lost }\end{array}$ & $\begin{array}{c}\text { \# times } \\
\text { defocusing } \\
\text { lens was lost }\end{array}$ \\
\hline First week & 2 & 0 & 2 \\
Second week & 8 & 2 & 10 \\
Third week & 12 & 3 & 15 \\
Fourth week & 13 & 3 & 16 \\
\hline Total & 35 & 8 & 43 \\
\hline
\end{tabular}

${ }^{\text {a Times defocusing lens was lost indicate the total of times spectacle frame }}$ was lost and times entire apparatus was lost.

TABLE 2: A comparison of the reported apparatus stability and the head-mounted spectacle frame.

\begin{tabular}{lcc}
\hline Compliance (\# times defocusing lens was lost) & $\leq 2$ & $\geq 3$ \\
\hline Our apparatus & $30 / 30$ & $0 / 30$ \\
\hline Head-mounted goggling apparatus [18] & $24 / 28$ & $4 / 28$ \\
\hline Glued to the fur [18] & $13 / 30$ & $17 / 30$
\end{tabular}

Our apparatus versus the head-mounted goggling apparatus, Fisher's Exact Test, $p=0.0482$.

Our apparatus versus the glued to the fur technique, Fisher's Exact Test, $p<$ 0.001 .

28 days later, the right eye of mice in experimental group showed a significant myopic shift compared to the left eye $\left(-4.90 \pm 2.17 \mathrm{D}\right.$; paired $t$-test $\left.p=1.69 \times 10^{-9}\right)$, but not found in the control group $(-0.90 \pm 1.37 \mathrm{D}$; paired $t$-test $p=$ 0.07). The axial length measurements using OCT (Table 3 ) showed no significant difference between the right and left eyes in both experimental $(0.004 \pm 0.034 \mathrm{~mm}$; paired $t$-test $p=0.58)$ and control group $(0.004 \pm 0.016 \mathrm{~mm}$; paired $t$ test $p=0.48$ ) at P28. After the induction period, the right eyes of mice in experimental group were significantly longer than the contralateral eyes $(0.025 \pm 0.037 \mathrm{~mm}$; paired $t$-test $p=0.002$ ), while no significant difference was found in the controls $(-0.013 \pm 0.031 \mathrm{~mm}$; paired $t$-test $p=0.22)$.

Postmortem evaluation showed general reactive hyperplasia of the skin and no obvious alteration of the skull underlying the screws. Histological examination revealed chronic inflammation of the hypoderm due to the foreign body reaction in the H\&E stained sections when compared with the normal controls, and the skull at the screw locations appeared healthy with no bony destruction (Figure 6).

\section{Discussion}

It is generally known that form deprivation and lens defocusing are the two classical methods for myopia induction in animal, and they were also reported to be used in the study of mouse myopia $[9,21]$. No matter which method is chosen, existing techniques for device attachment can be shared. In this study, we used the lens-induced myopia model for the reason of renewed interest that myopia progression in humans and animals can be altered through optical intervention $[5,22]$.

A literature survey showed that Tejedor and de la Villa made the first attempts by lid-suturing to induce deprivation myopia in mouse; however, it was not suitable for defocusing lens attachment [15]. Later, Schaeffel et al. introduced the method that goggles directly glued to the fur around the eye, and it was improved by Barathi et al. to be used in lensinduced myopia $[16,17]$. The method using glue was technically simple, but the device stability was not reliable enough and may lead to poor ocular health. Thus, Tkatchenko et al. reported a further refined technique, using a combination of glue and sutures [11]. Although the application of this method may not be restrained by mouse age and the effectiveness of myopia induction was reported to be good, no compliance data was shown. And our informal observations revealed that the stability has yet to be improved. Then some researchers used plastic collar to improve stability; however, it would inevitably affect the physical activity and whole health [11, 17]. Faulkner et al. developed a noncontact head-mounted goggle apparatus for the study of murine myopia [18]. It was reported to have satisfactory position stability and effective induction. However, the surgical procedures for the delicate device were quite complicated and time consuming and may lead to skull or brain injury. In addition, it was not suitable to be used in younger mouse with fragile skull, which may also make restriction on its popularity. Thus, it is still necessary to invent an easily operated, safe, and effective device for the myopia induction in mice.

Thus we developed the head-mounted spectacle frame and introduced a relatively easy-to-learn fixation method. Firstly, this device is lightweight (the nylon connector, spectacle frame, and defocusing lens amount to $0.50-0.60$ grams) and could enhance the compliance of spectacle frame wearing. Comparing with the head-mounted goggle apparatus, our research results demonstrated at least evenly matched position stability. In our study, there were still situations that indicated that the defocusing lens was not permanently fixed, and the ability to maintain device stability tended to decrease with extended induction time. Therefore, it was of great importance to tighten the screws when operating and to check the fitting situation carefully during the experimental period. Secondly, our device showed satisfactory safety. The body weight gradually increased during the experimental period, with no obvious difference with the reference values. With sutures fixation but not skull implantation, there were only chronic subdermal inflammations found at the screw locations, but no pathological change in the skull or adjacent brain. Thirdly, and perhaps most importantly, our device and method induced myopic shift and axial elongation in the experimental eyes. Literatures have shown that a $-10 \mathrm{D}$ lens fitted over the mouse eye could induce a refractive shift of $-13.03 \mathrm{D}$ in 46 days (P10-P56) under a 12:12 h light: dark cycle [16], and a -25 lens induced a $14.6 \mathrm{D}$ myopic shift in 21 days (P24-P45) under constant light [11]. In this study, the myopic shift of $4.90 \mathrm{D}$ in 28 days (P29-P56) is relatively small and is approximately equal to that reported by Faulkner et al. using a head-mounted goggling apparatus $(5.66 \mathrm{D}$ myopic shift in 14 days of form deprivation) [18]. Many 
TABLE 3: Results of retinoscopy refraction and axial length measurements.

\begin{tabular}{|c|c|c|c|c|c|}
\hline & \multirow{2}{*}{ Age } & \multicolumn{2}{|c|}{ Relative refraction (diopters) } & \multicolumn{2}{|c|}{ Axial length (mm) } \\
\hline & & OD-OS & $p$ value* & OD-OS & $p$ value $^{*}$ \\
\hline \multirow{2}{*}{ Experimental group } & P28 & $0.08 \pm 1.26$ & 0.753 & $0.004 \pm 0.034$ & 0.577 \\
\hline & P56 & $-4.90 \pm 2.17$ & $1.69 \times 10^{-9}$ & $0.025 \pm 0.037$ & 0.002 \\
\hline \multirow{2}{*}{ Control group } & P28 & $0.70 \pm 1.34$ & 0.132 & $0.004 \pm 0.016$ & 0.482 \\
\hline & P56 & $-0.90 \pm 1.37$ & 0.068 & $-0.013 \pm 0.031$ & 0.220 \\
\hline
\end{tabular}

Experimental group: $n=25$; control group: $n=10$.

OD-OS: values indicated the difference between the right and left eyes.

*Student's paired $t$-test.

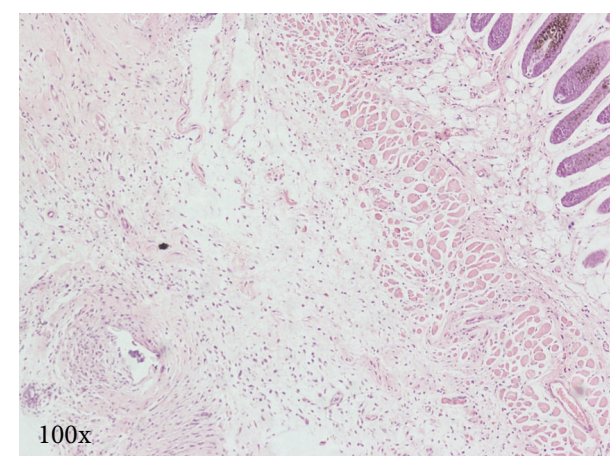

(a)

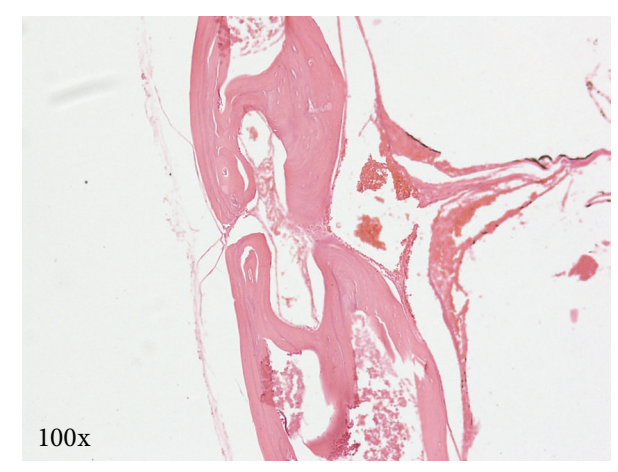

(b)

FIGURE 6: H\&E stained sections of the skin (a) and the skull (b) at the screw sites. Note the reactive hyperplasia of the subcutaneous tissue, and the absence of a glaucomatous reaction or destructive changes to the skull.

explanations may contribute to the reduced effectiveness, such as species variability of the mice, a later induction initiation, or others. In any case, the head-mounted spectacle frame apparatus is reliable and practicable. In addition, our device is economical and the surgical procedures are easy to learn, for only requiring commonly used microscopic suture materials but not professional equipment. This is also a beneficial aspect of our device and method in consideration of the popularization.

Some limitations should be considered in the current study. First, the physical dimensions of the one-piece frame were fixed in this study, which may not be compatible with mouse younger than P28. Thus frames with optimized physical dimensions adapting to younger mice during the susceptible period for myopia induction could substantially increase a future use of this device. Second, the introduced method is a rather invasive procedure, which requires recovery and infection and pain management. Thus design of inflammation and pain management may be necessary in further study. Third, although other reported methods and procedures were not easy to duplicate, it was still of significance to make comparison between these methods and our method at the same time, including effectiveness, security. And experiments along these lines are currently in progress in our laboratory.

In summary, we described a novel apparatus named the head-mounted spectacle frame apparatus and represented a simplification of the currently employed head fixation techniques for study of myopia in mouse. Our results demonstrated several advantages, including the improved compliance, effective myopia induction, and reliable security. The new method also has the potential to test form deprivation myopia. All of these features would likely prove useful in small animal models among investigators studying experimental myopia trials.

\section{Conflict of Interests}

The authors declare that there is no conflict of interests regarding the publication of this paper.

\section{Authors' Contribution}

Yangshun $\mathrm{Gu}$, Baisheng $\mathrm{Xu}$, and Bo Jiang conceived and designed the experiments. Yangshun Gu, Baisheng Xu, Chunfei Feng, Qin Wu, Yang $\mathrm{Ni}$, and Bo Jiang performed the experiments. Yangshun $\mathrm{Gu}$, Baisheng $\mathrm{Xu}$, Chunfei Feng, Nan Hong, and Bo Jiang analyzed the data. Qin Wu, Yang $\mathrm{Ni}$, Chixin Du, Peng Li, and Zhihua Ding contributed reagents/materials/analysis tools. Yangshun $\mathrm{Gu}$, Baisheng $\mathrm{Xu}$, and Bo Jiang wrote the paper. Yang Ni, Chixin Du, Peng Li, and Zhihua Ding designed the software used in OCT imaging analysis.

\section{Acknowledgments}

This work was supported by the National Natural Science Foundation of China (Grant nos. 81200717, 61475143, and 
61335003); the Specialized Research Fund for the Doctoral Program of Higher Education (Grant no. 20120101120027); Zhejiang Provincial Natural Science Foundation of China (LY14F050007). The authors are grateful to Jianxin Wang (Versolsolar Hangzhou Co., Ltd.) for advice and technical assistance on the design of head-mounted spectacle frame apparatus.

\section{References}

[1] I. G. Morgan, K. Ohno-Matsui, and S.-M. Saw, "Myopia," The Lancet, vol. 379, no. 9827, pp. 1739-1748, 2012.

[2] C.-T. Xu, S.-Q. Li, Y.-G. Lü, and B.-R. Pan, "Development of biomedical publications on ametropia research in PubMed from 1845 to 2010: a bibliometric analysis," International Journal of Ophthalmology, vol. 4, no. 1, pp. 1-7, 2011.

[3] T. R. Fricke, B. A. Holden, D. A. Wilson et al., "Global cost of correcting vision impairment from uncorrected refractive error," Bulletin of the World Health Organization, vol. 90, no. 10, pp. 728-738, 2012.

[4] W. N. Charman, "Myopia revisited, 2010-2015," Ophthalmic and Physiological Optics, vol. 35, no. 6, pp. 637-642, 2015.

[5] E. L. Smith III, L.-F. Hung, and B. Arumugam, "Visual regulation of refractive development: insights from animal studies," Eye, vol. 28, no. 2, pp. 180-188, 2014.

[6] M. H. Edwards, "Animal models of myopia. A review," Acta Ophthalmologica Scandinavica, vol. 74, no. 3, pp. 213-219, 1996.

[7] X. T. Zhou and J. Qu, "Selection of animal model in myopia research," Zhonghua Yan Ke Za Zhi, vol. 41, no. 6, pp. 486-487, 2005.

[8] W. Shen, M. Vijayan, and J. G. Sivak, "Inducing form-deprivation myopia in fish," Investigative Ophthalmology and Visual Science, vol. 46, no. 5, pp. 1797-1803, 2005.

[9] M. T. Pardue, R. A. Stone, and P. M. Iuvone, "Investigating mechanisms of myopia in mice," Experimental Eye Research, vol. 114, pp. 96-105, 2013.

[10] X. T. Zhou, J. Qu, and F. Lu, "Dose the mice is ideal animal myopia model?” Zhonghua Yan Ke Za Zhi, vol. 44, no. 7, pp. 584-586, 2008.

[11] T. V. Tkatchenko, Y. Shen, and A. V. Tkatchenko, "Mouse experimental myopia has features of primate myopia," Investigative Ophthalmology and Visual Science, vol. 51, no. 3, pp. 1297-1303, 2010.

[12] H. Park, C. C. Tan, A. Faulkner et al., "Retinal degeneration increases susceptibility to myopia in mice," Molecular Vision, vol. 19, pp. 2068-2079, 2013.

[13] R. Chakraborty, H. n. Park, A. M. Hanif, C. S. Sidhu, P. M. Iuvone, and M. T. Pardue, "ON pathway mutations increase susceptibility to form-deprivation myopia," Experimental Eye Research, vol. 137, pp. 79-83, 2015.

[14] M. T. Pardue, A. E. Faulkner, A. Fernandes et al., "High susceptibility to experimental myopia in a mouse model with a retinal on pathway defect," Investigative Ophthalmology and Visual Science, vol. 49, no. 2, pp. 706-712, 2008.

[15] J. Tejedor and P. de la Villa, "Refractive changes induced by form deprivation in the mouse eye," Investigative Ophthalmology and Visual Science, vol. 44, no. 1, pp. 32-36, 2003.

[16] V. A. Barathi, V. G. Boopathi, E. P. H. Yap, and R. W. Beuerman, "Two models of experimental myopia in the mouse," Vision Research, vol. 48, no. 7, pp. 904-916, 2008.
[17] F. Schaeffel, E. Burkhardt, H. C. Howland, and R. W. Williams, "Measurement of refractive state and deprivation myopia in two strains of mice," Optometry and Vision Science, vol. 81, no. 2, pp. 99-110, 2004.

[18] A. E. Faulkner, M. K. Kim, P. M. Iuvone, and M. T. Pardue, "Head-mounted goggles for murine form deprivation myopia," Journal of Neuroscience Methods, vol. 161, no. 1, pp. 96-100, 2007.

[19] Y. Ni, B. Xu, L. Wu et al., "Assessment of full-eye response to osmotic stress in mouse model in vivo using optical coherence tomography," Journal of Ophthalmology, vol. 2015, Article ID 568509, 8 pages, 2015.

[20] P. Li, Z. Ding, Y. Ni et al., "Visualization of the ocular pulse in the anterior chamber of the mouse eye in vivo using phasesensitive optical coherence tomography," Journal of Biomedical Optics, vol. 19, no. 9, Article ID 090502, 2014.

[21] I. G. Morgan, R. S. Ashby, and D. L. Nickla, "Form deprivation and lens-induced myopia: are they different?" Ophthalmic and Physiological Optics, vol. 33, no. 3, pp. 355-361, 2013.

[22] P. R. Sankaridurg and B. A. Holden, "Practical applications to modify and control the development of ametropia," Eye, vol. 28, no. 2, pp. 134-141, 2014. 


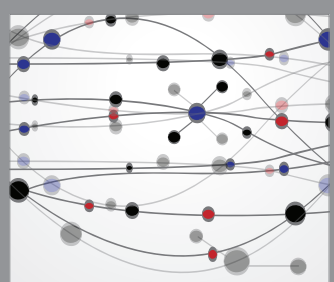

The Scientific World Journal
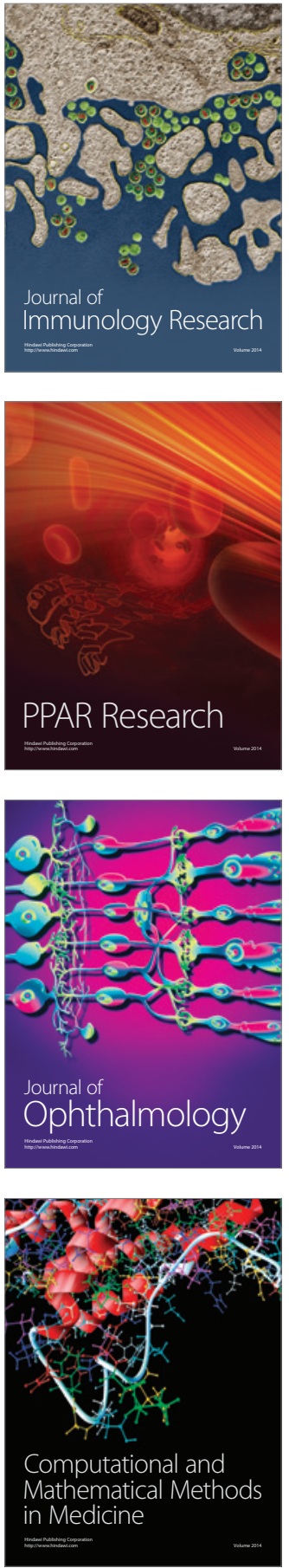

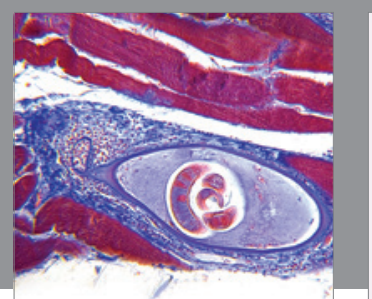

Gastroenterology Research and Practice

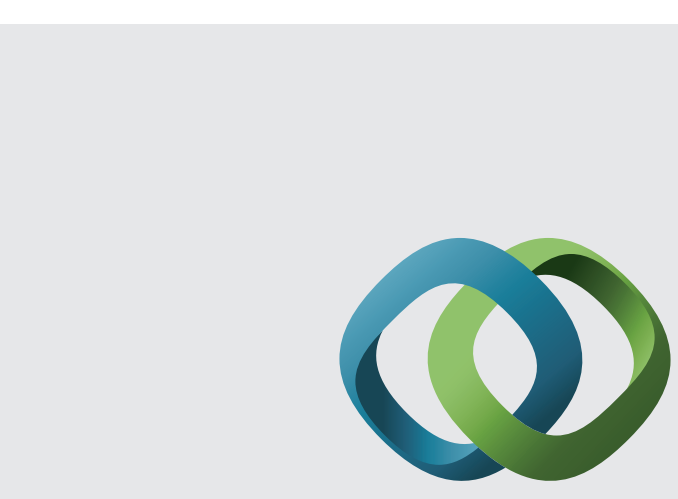

\section{Hindawi}

Submit your manuscripts at

http://www.hindawi.com
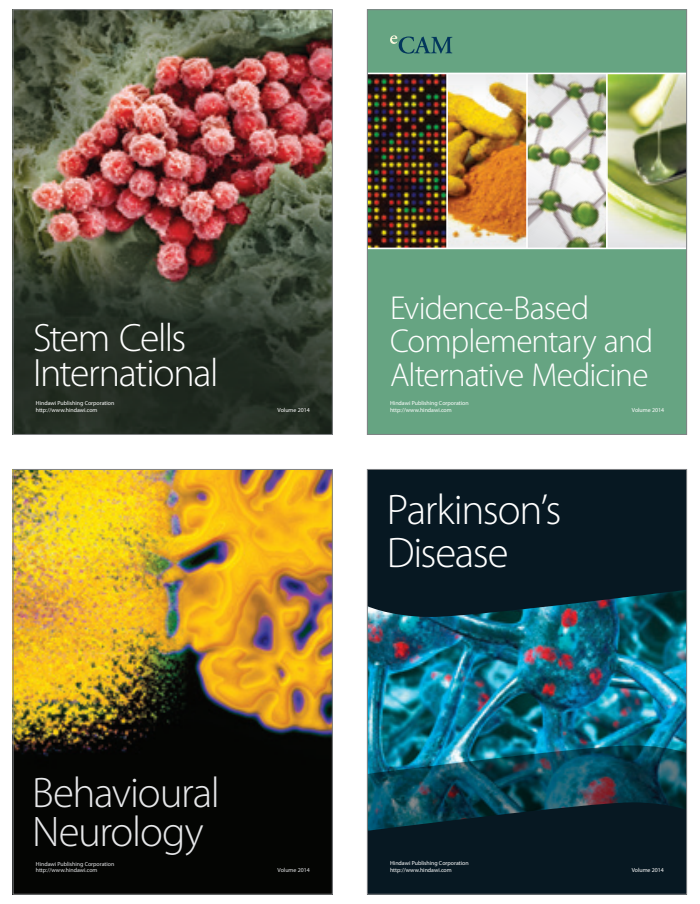
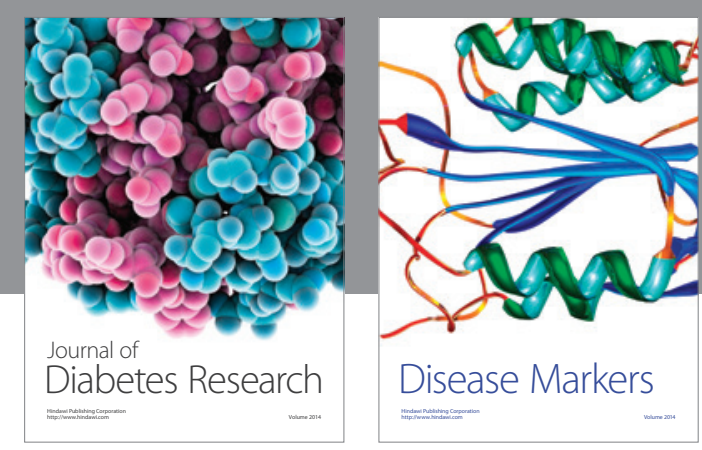

Disease Markers
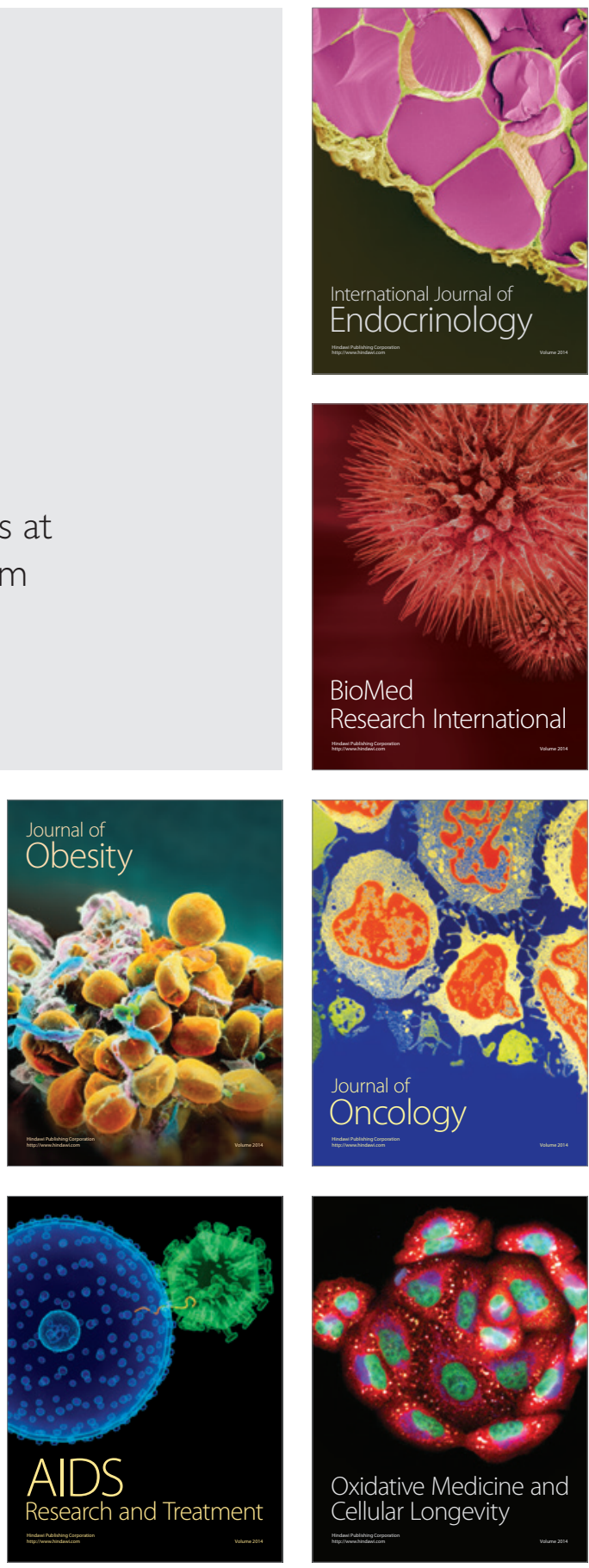